\title{
Construction, Installation and Commissioning of a High-Efficiency and High-Resolution Straw Tube Tracker for the LHCb experiment ${ }^{1}$
}

\author{
F.Jansen ${ }^{\mathrm{a}, *}$ \\ on behalf of the LHCb Outer Tracker collaboration \\ ${ }^{a}$ Nikhef, Science Park 105, 1098XG Amsterdam, the Netherlands
}

\begin{abstract}
The LHCb experiment is a single arm spectrometer, designed to study $\mathrm{CP}$ violation in $B$-decays and search for new physics in rare $B$-decays at the Large Hadron Collider (LHC). In such studies it is crucial to accurately and efficiently track the charged decay products in the high-density environment of the LHC. For this, the Outer Tracker (OT) was constructed, consisting of 55,000 straw tubes and covering an area of $360 \mathrm{~m}^{2}$ of module layers. The detector is foreseen to operate under large particle rates, up to $100 \mathrm{kHz} / \mathrm{cm}$ per straw in the region closest to the beam.

Before and during installation the detector modules and Front End (FE) electronics have been extensively tested. The detector has been installed and commissioned with the help of cosmic ray events.
\end{abstract}

Key words: Tracking detectors, Gas detectors, Straw tubes

PACS: 29.40.Cs, 29.40.Gx, 07.05.Fb, 07.77.Ka, 12.15.Hh

\section{Introduction}

The LHCb experiment [1] has been tailored for the study of $B$-hadron physics. It will precisely measure the Charge-Parity asymmetry in $B$-meson decays and it will probe physics beyond the Standard Model through rare $B$-decays.

The LHC will act as a $B$ factory with a large b-quark production rate $\left(\sigma_{b \bar{b}} \approx 500 \mu \mathrm{m}\right)$, where the $B$-hadrons are predominantly produced in the forward or backward direction. For this, the LHCb experiment has been designed as a one-arm forward spectrometer, with an angular acceptance of 10-300 mrad and will run at the luminosity of $2 \times 10^{32} \mathrm{~cm}^{-2} \mathrm{~s}^{-1}$, where approximately $10^{12} \mathrm{~b} \bar{b} /$ year are expected.

The Outer Tracker and Inner Tracker form the tracking system upstream of the LHCb magnet [1]. The Outer Tracker is a straw tube detector that covers the LHCb acceptance except for a small region around the beam pipe which is covered by the silicon strip based Inner Tracker detector.

In order to have efficient $B$-hadron reconstruction, the tracking efficiency of charged particles should be excellent $(\sim 100 \%)$. This in turn requires the straw occupancy to be less than $10 \%$. A precise mass reconstruction calls for a momentum resolution on charged particles of better than $0.5 \%$, or a spatial resolution less than $200 \mu \mathrm{m}$. Furthermore, the Outer Tracker has to cope with the $25 \mathrm{~ns}$ bunch crossing cycle of the LHC.

\footnotetext{
${ }^{*}$ Corresponding author

Email addresses: fabianj@nikhef .nl (F.Jansen)

${ }^{1}$ Presented at the $11^{\text {th }}$ Pisa meeting on advanced detectors, Elba, Italy $25-$ 29 May 2009.
}

\section{Outer Tracker module design and production}

The Outer Tracker consists of 3 tracking stations. The stations are positioned $0.5 \mathrm{~m}$ apart along the beam line and each station has 4 layers of detection modules, which are mounted vertically on support frames. The middle two layers of each station have a small stereo angle of $+5^{0}$ and $-5^{0}$.

Every detection layer consists of 18 detector modules mounted side by side. The central 4 modules of a layer have a gap in the middle, that allows for the beam pipe and for a region of too high occupancy, which is covered by the Inner Tracker.

There are 4 independent support frames (C-frames) per station, 2 on either side of the beam pipe, so that each C-frame supports 9 detection modules on its front side and 9 modules on its back side. The $\mathrm{C}$-frames are supported on a rail, to allow extraction in and out of the LHCb acceptance. Figure 1a shows a schematic overview of a C-frame.

The $\mathrm{C}$-frames distribute the gas to the detector modules and distribute cooling, high voltage, slow and fast control, etc. to the FE electronics.

\subsection{Module design}

A sketch of the module design is shown in figure $1 \mathrm{~b}$. The module dimensions are $490 \times 34 \mathrm{~cm}^{2}$ and one module consists of 2 staggered layers of 64 straw tubes sandwiched between panels. The panels are Rohacell between carbon fiber for rigidity, with a layer of kapton foil for gas tightness and a layer of aluminum for shielding and common grounding. Electrically the modules are split in an upper and lower section, that are individually read out by FE electronics. 


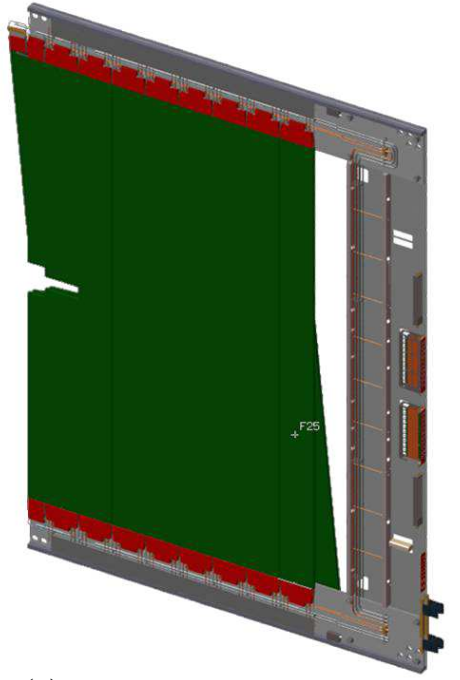

(a)

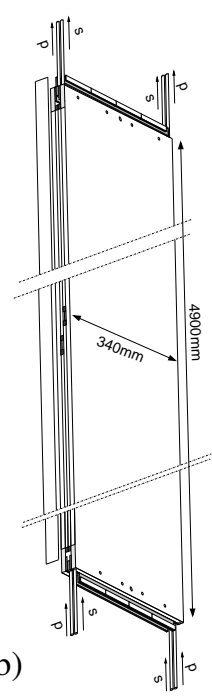

Figure 1: (a) An OT C-frame: A rigid aluminum structure distributes gas, cooling and electrical and optical connections to the detection modules and FE electronics. Nine modules are mounted on either side of the frame. (b) An OT detection module: 2 staggered layers of 64 straw tubes between rigid panels, with gas connection and electrical feed through boards on both ends.

The straws are $5 \mathrm{~mm}$ in diameter. At operation they are flushed with a $\operatorname{Ar}(70 \%) / \mathrm{CO}_{2}(30 \%)$ gas mixture, while the anode wires are set at a high voltage of $1550 \mathrm{~V}$. The cathode consists of aluminum laminated kapton foil on carbon doped kapton-XC foil. A $25 \mu \mathrm{m}$ gold plated tungsten wire in the center of the straw serves as anode.

The material budget of one module is $\sim 0.74 \% \mathrm{X}_{0}$ [2].

\subsection{Module production}

In 2004-2005 the OT modules were constructed in Amsterdam, Heidelberg and Warsaw whereas all support panels were built in Krakow [3]. During production the anode wire tension, wire position and dark currents were monitored. The tension of anode wires is set at $70 \mathrm{~g}$ and the overall variation in wire tension is less than $5 \mathrm{~g}$. The position of the wire with respect to the nominal deviates no more than $100 \mu \mathrm{m}$. And dark currents in air were measured to be smaller than $10 \mathrm{nA}$ at $1600 \mathrm{~V}$ high voltage.

After completion of a module, it was tested for gas tightness and dark currents, while the module was filled with the $\mathrm{Ar} / \mathrm{CO}_{2}$ gas mixture. The leak rates were found to be less than $1 \mathrm{mbar} /$ minute and leakage currents below $10 \mathrm{nA}$ at $1600 \mathrm{~V}$.

In addition, the response of every channel was checked with a radioactive source. An example of a full scan with a ${ }^{90} \mathrm{Sr}$ source of a module is shown in figure 2. During the scan the source irradiates the full width of a module and at every $\mathrm{cm}$ of its length, thereby obtaining a response profile for the detector modules.

After module production $0.06 \%$ of the channels were found faulty and are disconnected and $0.07 \%$ of the channels were

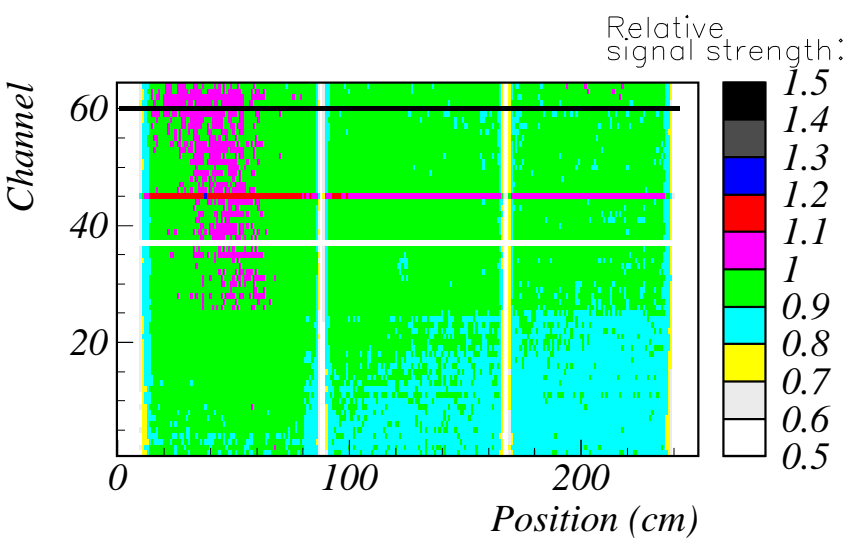

Figure 2: Response profile of module S1L65A, as determined by a scan with a ${ }^{90} \mathrm{Sr}$ source, directly after production. Channel 37 is a disconnected straw, while channel 60 is a noisy straw. The vertical lines at $\sim 85 \mathrm{~cm}$ and $\sim 65 \mathrm{~cm}$ correspond to wire support elements.

found with high dark currents, which often are cured by HV training.

\subsection{Front End electronics production}

All OT detection modules are electrically split into an upper and a lower half. The halves are individually read out by FE electronics boxes [4], that connects to feed-through boards that are built into the module.

Every box houses 4 types of circuit boards that are mounted onto an aluminum support frame and are shielded by aluminum covers. High Voltage circuit boards connect to the anode wires of a module. The amplifier-discriminator (ASDBLR) receives signals from a HV board and discriminates them against threshold. The binary signals from the ASDBLR are given a time stamp by the OTIS time-to-digital converter. And the GOL circuit board (Gigabit Optical Link) collects the timing data of all 128 channels and ships it off-detector via single optical fiber.

In the period 2005-2008 the FE electronics boxes were assembled in Amsterdam. Before assembly all electronics components were tested and selected individually, while after assembly the boxes were tested with a dedicated setup [5].

Details about the design and testing of the OT FE electronics can be found elsewhere [6].

\section{Outer tracker installation and commissioning}

\subsection{Test beam results}

In the beginning of 2005 four OT modules, together with the final prototype of the FE electronics, were subjected to a beam test at Desy [7]. The beam test with $6 \mathrm{GeV}$ electrons allowed to determine the efficiency, resolution and noise and cross talk as a function of the supplied high voltage, the amplifier threshold and the position along the straws. The resolution was determined both by using a silicon telescope for position reference, and with OT standalone tracking. 

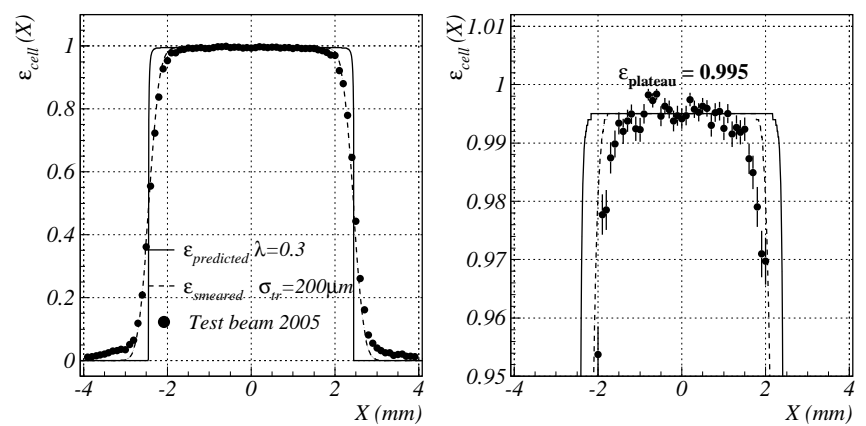

Figure 3: (a) Straw tube efficiency profile, as obtained by the beam test. (b) A zoom in of the plateau region reveals an efficiency of $>99 \%$ in the region $\pm 1.6 \mathrm{~mm}$.

The beam test demonstrated an efficiency of $\sim 98 \%$, a position resolution better than $200 \mu \mathrm{m}$ and a noise rate and cross talk level below $10 \mathrm{kHz} /$ wire and $4 \%$ respectively, measured at $1550 \mathrm{~V}$ and with an amplifier threshold of $800 \mathrm{mV}$ (corresponding to about $4 \mathrm{fC}$ ). Furthermore, a first TR-relation and efficiency profile along the straw diameter were derived. The efficiency profile is shown in figure 3 .

\subsection{Installation}

In 2006-2007 the OT detection modules were installed on their support frames (C-frames) inside the LHCb cavern, after which the support frames were inserted into the $\mathrm{LHCb}$ detector [8].

Before assembly of the modules, the C-frames themselves were tested for gas tightness of the gas distribution system, high voltage leakage currents and electrical and optical connections. The gas tightness of the distribution system was $<1 \mathrm{mbar} /$ minute at $10 \mathrm{mbar} \mathrm{CO}_{2}$, leakage currents of the $\mathrm{HV}$ cables were below $10 \mathrm{nA}(68 \%$ within $1 \mathrm{nA})$, all electrical connections satisfied specifications and the optical fiber attenuation less than $30 \mathrm{dBm}$.

After the modules were assembled into a C-frame, they were again tested for gas tightness and high voltage leakage currents. The module leak rate was measured to be less than $1 \mathrm{mbar} /$ minute and matching production values, while the high voltage leakage currents were $<100 \mathrm{nA}$ after 3 minutes, while the modules were flushing with $\mathrm{CO}_{2}$ and being supplied with $1800 \mathrm{~V}$ high voltage.

Furthermore, more than half of all the straws of the Outer Tracker were individually tested in situ for their functionality with a ${ }^{55} \mathrm{Fe}$ source. An example of the test results for a series of 9 module-layers of one C-frame are shown in figure 4. In total, 13 irresponsive channels were found, out of which eleven had been known at production.

In 2008 all FE electronics boxes were installed onto the Outer Tracker detector, resulting in an Outer Tracker with fully operational FE boxes.

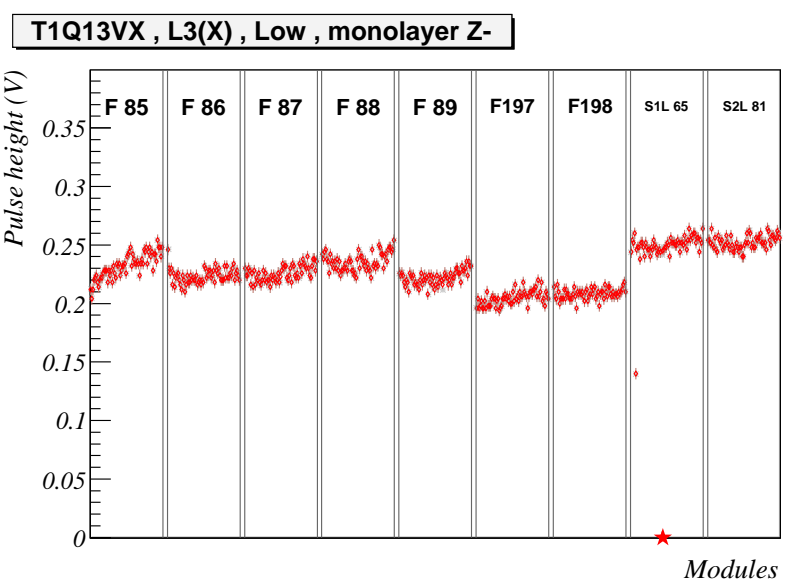

Figure 4: Response to a ${ }^{55} \mathrm{Fe}$ source of straws of C-frame T1-Q13-VX, after installation. The irresponsive straw and the straw with low average pulse height in module S1L65 correspond to the disconnected and noisy straw respectively of figure 2 .

\subsection{Commissioning with cosmic ray events}

During the last year the Outer Tracker has been taking data in the global LHCb commissioning scheme. Different types of data have been taken with different goals in mind. Random triggers are used to establish noise levels and debug the FE electronics. The Outer Tracker includes the possibility to provide the FE electronics with test pulses, which are used to reestablish the threshold characteristics, timing behavior, etc. of the the FE electronics boxes.

Furthermore, data taken from cosmic ray events are used for $\mathrm{t}_{0}$ calibration, TR calibration, and detector alignment. The readout of a cosmic ray event is triggered by the $\mathrm{LHCb}$ calorimeter, which is placed $3 \mathrm{~m}$ upstream of the last OT station. An initial geometrical survey using theodolites has determined the positions of the C-frames with an accuracy of $0.5 \mathrm{~mm}$.

To retrieve the drift time, the measured time of a cosmic ray in a straw is corrected for time of flight, wire propagation time and whether the cosmic muon passed the Outer Tracker before or after it was triggered by the calorimeter (upstream and downstream events respectively). The retrieved drift times and drift distances however include uncertainties in the $\mathrm{t}_{0}$ offsets, detector alignment and TR-relation.

These uncertainties are obtained from the cosmic ray events by iterative algorithms using the residuals of straw hits versus fitted track positions [9].

Cosmic ray events determine the $t_{0}$ of the OT FE electronics boxes as shown in figure 5 . The variation in time offset between FE electronics boxes is less than $3 \mathrm{~ns}$.

The obtained TR relation is shown in figure 6 . The figure shows good agreement between the relation as obtained with cosmic ray events and the one measured with the beam test.

Figure 7 shows the relative alignment of each OT C-frames, as determined with cosmic ray events. The alignment procedure shows that the $\mathrm{C}$-frames are placed at the nominal position within $1 \mathrm{~mm}$. These results are consistent with the geometrical survey, which were accurate to $0.5 \mathrm{~mm}$. 


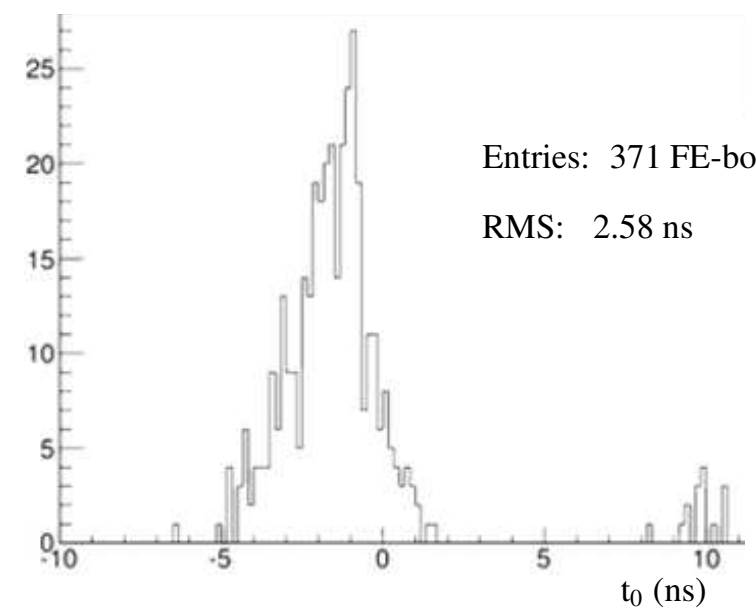

Figure 5: The $t_{0}$ offsets of the OT FE electronics boxes, as obtained with cosmic ray events. The $t_{0}$ at $\sim 10 \mathrm{~ns}$ are due to faulty CLK fan-outs, which have been replaced.

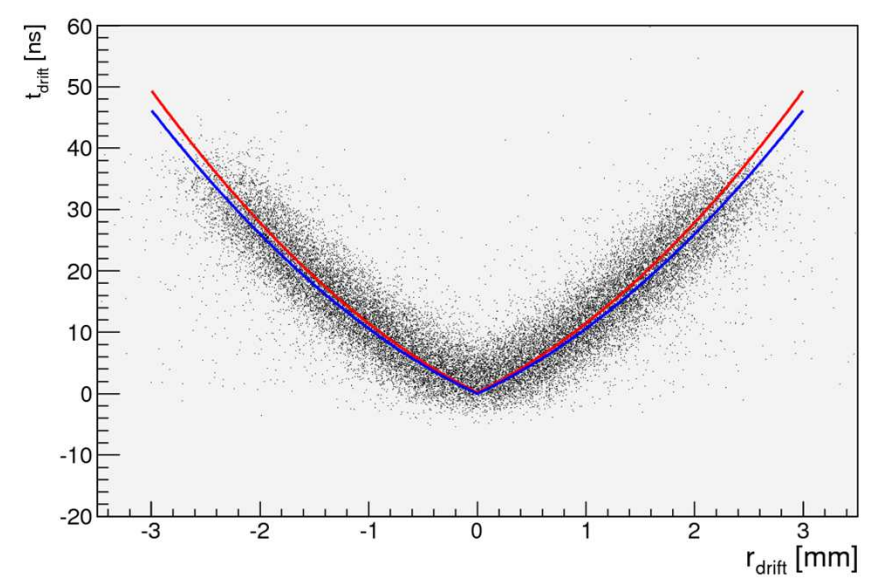

Figure 6: The relation between drift time and minimum drift distance (TR relation), as obtained with cosmic ray events. The upper red line is a fit to the data points. The lower blue line is the TR relation as fitted to test beam data.

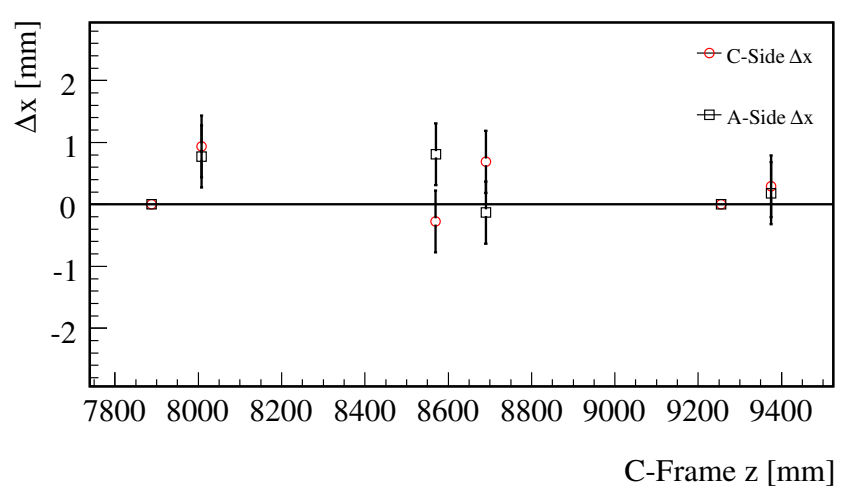

Figure 7: Relative deviation from nominal of the horizontal x-positions of the OT C-frames, determined from cosmic ray events. The $1^{\text {st }}$ and $5^{\text {th }}$ pair of Cframes are used as reference, which results in zero deviation by construction. The error bar indicates the accuracy of the geometrical survey.

\section{Conclusion}

$\mathrm{LHCb}$ is an experiment tailored for the study of $B$-hadron physics. The experiment will be ready to collect data with its full detector when the LHC turns on. The CP asymmetry in nature will be tested with the decays of $B$-mesons, while physics beyond the Standard Model will be probed with rare $B$-hadron decays.

For this, the tracking system performance is of crucial importance. The Outer Tracker is a high-efficiency and highresolution straw tube detector. All OT detection modules and FE electronics have been constructed and have been extensively tested, with quality assurance at every production step.

A beam test at Desy has confirmed that the OT modules and FE electronics meet their requirements.

All OT modules and FE electronics have been installed onto their support frames in situ and retested as a whole. All frames have been installed into the LHCb detector and the Outer Tracker detector has been commissioned with the help of cosmic ray events.

\section{References}

[1] LHCb Coll., The LHCb Detector at the LHC, 2008, JINST, 3 S08005

[2] J. Nardullli, N. Tuning, A Study of the Material in an Outer Tracker Module, , 2004, CERN-LHCb-2004-114

[3] N. Tuning et al., Outer Tracker Module Production at NIKHEF - Quality Assurance, 2004, CERN-LHCb-2004-078

[4] A. Berkien et al., The LHCb Outer Tracker Front End Electronics, 2005, ERN-LHCb-2005-025

[5] E. Simioni et al., OT FE-Box Test Procedures, 2007, CERN-LHCb-2007122

[6] F. Jansen et al., The Front End Electronics of the LHCb Straw Tube Tracker, 2009, Published in this issue

[7] G. van Apeldoorn et al., Beam Tests of Final Modules and Electronics of the LHCb Outer Tracker in 2005, 2005, CERN-LHCb-2005-076

[8] F. Jansen et al., Test and Assembly of the Outer Tracker $C$-frames and Detector Modules at Installation, 2008, CERN-LHCb-2008-064

[9] L. Nicolas et al., Alignment of LHCb tracking stations with tracks fitted with a Kalman filter , 2008, CERN-LHCb-2008-066 